\title{
Wie sagen wir den Verlauf der IPF voraus?
}

\begin{abstract}
Hintergrund u. Fragestellung: Der vor Kurzem etablierte Gender-Age-Physiology-(GAP-)Index aus Geschlecht, Alter und physiologischen Messparametern (Vital-, Diffussionskapazität) ist ein zuverlässiger Prädiktor für den Verlauf einer idiopathischen Lungenfibrose (IPF), d.h. für die Mortalitätsvorhersage, und wird von vielen als "Staging" der IPF bezeichnet [1]. Jedoch wurden jüngst mehrere longitudinale Variablen als weitere Vorhersageparameter etabliert. Ziel war es daher, den GAP-Index mit anderen bei der Erkrankung essenziellen Parametern zu verbinden, um den Verlauf individueller vorhersagen zu können.
\end{abstract}

Patienten u. Methoden: 1109 IPF-Patienten wurden analysiert, um den GAP-Index und weitere wichtige Parameter zu testen.

Ergebnisse: Die Kombination des GAP-Index mit den longitudinalen Parametern Hospitalisation aus respiratorischen Gründen und Lungenfunktions-

\section{Originalie}

Brett Ley, Williamson Z Bradford et al. Unified baseline and longitudinal mortality prediction in idiopathic pulmonary fibrosis. Eur Respir J 2015; 45: 1374-81. verlauf (FVC) über 24 Wochen konnte die prädiktive Wertigkeit des GAP-Index stark bessern.

Schlussfolgerungen: Der erweiterte longitudinale GAPIndex kann als guter indivi-

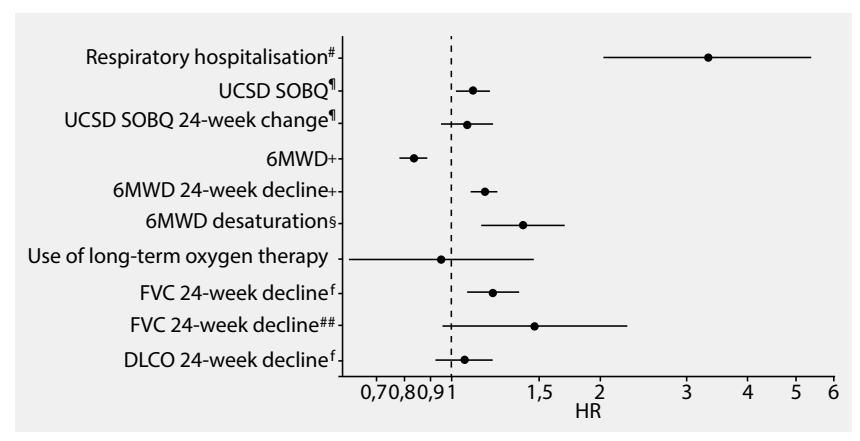

1 ssoziation individueller Variablen mit der Mortalität nach Adjustierung auf Geschlecht, Alter u. physiol. Paramter (GAP). UCSDSOBQ: University of California San Diego Shortness of Breath Questionnaire; 6MWD: 6-min walking distance; 6MWT: 6-min walk test; FVC:

Forced Vital Capacity; DLCO: Diffusionskapazität der Lunge für CO; HR: Hazard Ratio. \#: in den 24 Wochen davor; g: pro 10 Units; +: pro $50 \mathrm{~m}$; s: per 5\% Abnahme; f: continuous, per 5\%; \#\#: binary $>10 \%$.

dueller Vorhersageparameter der IPF-Mortalität genutzt werden und eignet sich womöglich für Studien und die tägliche Betreuung von IPF-Patienten. Aber er muss weiter validiert und auf seinen Vorhersagewert bezüglich akuter IPF-Exazerbationen geprüft werden.

\section{- Kommentar von Prof. Dr. med. Michael Kreuter}

\section{Wichtiges Ziel bei IPF: Respiratorische Verschlechterungen verhindern}

Schon länger schwelt die Diskussion, wie man den Verlauf bzw. die Mortalität der IPF am besten vorhersagen könnte - und dies nicht nur unter Studienbedingungen, sondern v.a. in der täglichen Arbeit mit IPF-Patienten. Mittlerweile hat sich die Bestimmung der Vitalkapazität als gutes Messinstrument zur Verlaufsbeurteilung durchgesetzt [2] und ist von der FDA als solches für Studien anerkannt worden [3]. Mittlerweile wurden aber auch weitere Parameter, die die Mortalität beeinflussen, etabliert. Vor allem gilt die Krankenhausaufnahme aus respiratorischen Gründen als einer der wichtigsten Parameter, die den Verlauf der IPF bzw. ihr Mortalitätsrisiko bestimmen [4]. Der klinische Alltag zeigt, dass eine respiratorische Verschlechterung aus jedweden Gründen, d.h. sowohl Pneumonie als auch akute Exazerbation oder andere, einen deutlich negativen Effekt für den IPF-Patienten hat. Hinzu kommt, dass dies nicht nur bei IPF sondern, wie eine jüngste Interimsanalyse des deutschen EXCITING-ILD-Registers zeigt, bei allen ILD-Patienten sehr häufig ist [5]. Daher muss es uns ein wichtiges Anliegen sein, nicht nur den Erhalt der Lungenfunktion positiv zu beeinflussen. Auch pulmonalen Infekten und anderen Gründen einer respiratorischen Verschlechterung, die zur Hospitalisierung führen, muss möglichst vorgebeugt werden. Gerade in der nun beginnenden Influenzasaison ist dies ein Appell an uns alle, an Impfungen zu denken.

\section{Literatur:}

1. Ley B, Ryerson CJ, Vittinghoff E et al. Ann Intern Med 2012; 156: 684-91.

2. Behr J, Bonella F, Bonnet R et.al U. Positionspapier zur Bedeutung der forcierten Vitalkapazität für Patienten mit idiopathischer Lungenfibrose (IPF). Pneumologie, im Druck, 2015

3. Karimi-Shah, B.A., Chowdhury B.A. N Engl J Med 2015; 372:1189-1191

4. Durheim MT et al. Lancet Respir Med 2015; 3: 388-96.

5. Kreuter M, Herth FJF et al. Interim analysis of the EXCITING-ILD registry, ERS congress 2015

Prof. Dr. med. Michael Kreuter Dr. med. Claudia Bauer

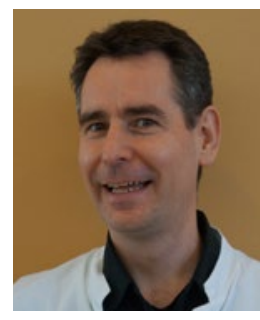

Pneumologie u. Beatmungsmedizin, Zentrum für interstitielle u. seltene Lungenerkrankungen, Thoraxklinik, Uniklinikum Heidelberg sowie Zentrum für Translationale Lungenforschung, Heidelberg (TLRC), Mitglied des Dtsch. Zentrums für Lungenforschung, Amalienstr. 5, 69126 Heidelberg, kreuter@uni-heidelberg.de 